\title{
Las Tecnologías de la Información y la Comunicación: Alternativa para la enseñanza de la Estadística Inferencial en la Educación Superior
}

\author{
DOI: https://doi.org/10.33262/ap.v3i3.1.75
}

\begin{abstract}
(c) (i) ()
Information and Communication Technologies: Alternative for the teaching of Inferential Statistics in Higher Education
\end{abstract}

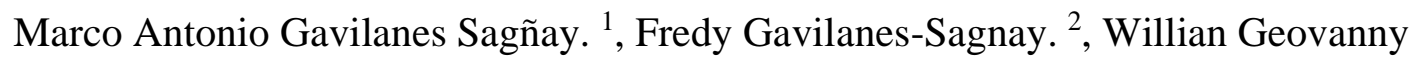
Yanza Chávez. ${ }^{3}$ \& Nathalie Azucena Chávez Granados. ${ }^{4}$

\section{Summary.}

Introduction. The improvement of the teaching-learning process in Higher Education continues to be a point of constant reflection. The teaching of Statistics given its characteristics is a subject of recurrent analysis, debate and reflection. Objective. Reflect on the importance of Information and Communication Technologies, as a timely alternative for optimizing the teaching-learning process of Statistics. Methodology. The methodology used was descriptive, documentary, based on the Systematic Review of Literature and the use of theoretical methods that favored the review and analysis of the preceding documentary information. Results. 1. The high utility that the use of Information and Communication Technologies has in the teaching-learning process in Higher Education is confirmed, with particular interest in the teaching of Statistics. 2. It is evident that despite the progress made, it is still necessary to develop training alternatives aimed at teachers to improve the teaching-learning process of Statistics through Information and Communication Technologies. 3. A transformative solution for

\footnotetext{
${ }^{1}$ Escuela Superior Politécnica de Chimborazo, Facultad de Administración de Empresas, Riobamba, Chimborazo, Ecuador, marco.gavilanes@espoch.edu.ec, https://orcid.org/0000-0002-7470-3732

2 Departamento de Ciencias de la Computación, Universidad de las Fuerzas Armadas ESPE sede Santo Domingo, Santo Domingo, Ecuador, fmgavilanes1 @espe.edu.ec, https://orcid.org/0000-0001-5494-374X

${ }^{3}$ Escuela Superior Politécnica de Chimborazo, Facultad de Administración de Empresas, Chimborazo Ecuador,willianyanza@hotmail.com, https://orcid.org/0000-0002-9688-7309

4 Investigador Independiente, Riobamba, Chimborazo, Ecuador, nathaliechavezg@gmail.com, https://orcid.org/0000-0002-4764-9160
} 
the teaching learning of Statistics can be the creation of interactive software. It is recommended that the solution proposal be established on the development of five phases: Diagnosis, Planning and Selection, Execution, Assessment and Generalization. Conclusions. The advantages of the applicability of Information and Communication Technologies in the teaching-learning process of Statistics have been substantiated; however, the necessary standards have not yet been achieved. It is necessary to implement strategies that favor the development of competencies in both teachers and students so that they can apply Information and Communication Technologies in the teachinglearning process of Statistics, as a timely alternative for the achievement of learning significant.

Keywords: Information and Communication Technologies, Statistics, teaching-learning process

\section{Resumen}

Introducción. El perfeccionamiento del proceso de enseñanza-aprendizaje en la Educación Superior, sigue siendo un punto de reflexión constante. La enseñanza de la Estadística dada sus características es un tema de recurrente análisis, debate y reflexión. Objetivo. Reflexionar sobre la importancia de las Tecnologías de la Información y la Comunicación, como una alternativa oportuna para la optimización del proceso de enseñanza-aprendizaje de la Estadística. Metodología. La metodología utilizada fue de tipo descriptiva, documental, basada en la Revisión Sistemática de Literatura y la utilización de métodos teóricos que favorecieron la revisión y análisis de la información documental precedente. Resultados. 1. Se confirma la alta utilidad que tiene en el proceso de enseñanza-aprendizaje en la Educación Superior el empleo de las Tecnologías de la Información y la Comunicación, con particular interés en la enseñanza de la Estadística. 2. Es evidente que a pesar de los avances logrados, aún se requiere desarrollar alternativas de capacitación direccionadas a los docentes para el perfeccionamiento del proceso de enseñanza- aprendizaje de la Estadística mediante las Tecnologías de la Información y la Comunicación. 3. Una solución transformadora para la enseñanza- aprendizaje de la Estadística puede ser la creación de software interactivo.4. Es recomendable que la propuesta de solución se establezca sobre el desarrollo de cinco fases: Diagnóstico, Planificación y Selección, Ejecución, Valoración y Generalización. Conclusiones. Ha quedado fundamentado las ventajas de la aplicabilidad de las Tecnologías de la Información y la Comunicación en el proceso de enseñanza-aprendizaje de la Estadística, sin embargo aún no se logran alcanzar los estándares necesarios. Se requiere implementar estrategias que favorezcan el desarrollo de competencias tanto en los docentes como en los estudiantes para que puedan aplicar las Tecnologías de la Información y la Comunicación en el proceso de enseñanza-aprendizaje de la Estadística, como una alternativa oportuna para el logro de aprendizajes significativos.

Palabras claves: Tecnologías de la Información y la Comunicación, Estadística, proceso de enseñanza-aprendizaje. 


\section{Introducción}

El desarrollo científico-tecnológico alcanzado en los últimos años ha proporcionado la introducción progresiva y ascendente de las Tecnologías de la Información y las Comunicaciones (TISc), como componente esencial dentro del contexto educativo en los diferentes niveles de enseñanza. En este ámbito se coincide con los referentes teóricos encontrados, en relación a que su empleo ha venido facilitando la dinamización del proceso de enseñanza-aprendizaje en los diferentes niveles de enseñanzas, carreras y asignaturas, influyendo positivamente en el pensamiento científico reflexivo de los estudiantes, así como en la integración de la teoría con la práctica.

En este contexto la enseñanza de la Estadística, dada sus propias características es un tema de recurrente análisis, debate y reflexión; precisamente por la necesidad de buscar alternativas que hagan que este proceso sea lo más efectivo posible. Particular interés en este orden de ideas tienen los aportes de Faustino, \& Pérez, (2013), al sostener que la utilización de las Tecnologías de la Información y las Comunicaciones en la enseñanza de la Estadística en la Educación Superior, han jugado un rol fundamental al facilitar dotar a los estudiantes de las competencias requeridas para su aprendizaje, unido a la apropiación de sólidos conocimientos en vínculo directo con el desarrollo histórico social y científico tecnológico de cada país.

Ahora bien, es importante comprender que para que se dé un adecuado proceso de enseñanza-aprendizaje de la Estadística en la Educación Superior, deben integrarse los programas informáticos al proceso formativo desde los primeros momentos. Recordemos que la Estadística forma parte del grupo de asignaturas que integran el perfil aplicativoinvestigativo de cualquier carrera, de ahí que sea necesario para optimizar su aprendizaje fomentar el manejo de los recursos informáticos, la formación de conceptos, el desarrollo del pensamiento epistemológico estadístico, conjuntamente con la capacidad de reflexión, de análisis crítico y la búsqueda de soluciones a los diferentes problemas. Ello permitirá que el estudiante logre desarrollar nuevas habilidades, en la misma medida que va aprendiendo a modelar las posibles soluciones basadas en la veracidad que ofrece la comprobación estadística.

Por supuesto que lograr lo anterior se convierte en un constante desafío; entre otros aspectos porque no siempre se cuenta con todos los recursos tecnológicos para su desarrollo y aprendizaje, unido a las limitaciones existentes en los Docentes Investigadores para el manejo apropiado de la Estadística y la falta de creatividad en la búsqueda de alternativas didácticas. Un ejemplo de lo señalado está justamente en el uso de las Tecnologías de la Información y la Comunicación para facilitar el proceso de enseñanza-aprendizaje en esta área; como es lógico pensar todo ello incide en las metodologías de enseñanza y en su aplicabilidad.

Otro de los aspectos que intervienen está relacionado con el propio proceso formativo; precisamente porque no siempre se reconoce en toda su dimensión la importancia y aplicabilidad de esta asignatura en las mallas curriculares de las diferentes carreras. Motivados por lo anteriormente señalado cabe preguntarnos ¿Cómo perfeccionar el 
proceso de enseñanza-aprendizaje de la Estadística en la Educación Superior? Partiendo de la interrogante formulada el presente artículo tiene como Objetivo: Reflexionar sobre la importancia de las Tecnologías de la Información y la Comunicación, como una alternativa oportuna para la optimización del proceso de enseñanza-aprendizaje de la Estadística en el contexto de la Educación Superior.

\section{Las Tecnologías de la Información y la Comunicación y su relación con la enseñanza -aprendizaje de la Estadística en la Educación Superior. De lo general a lo particular.}

La introducción de las Tecnologías de la Información y la Comunicación ha provocado una renovación progresiva y ascendente, de vital importancia en el contexto educativo en los diferentes países y niveles de enseñanza; su aplicación ha permitido que el proceso de enseñanza-aprendizaje se beneficie ostensiblemente, pasando de ser una invención aislada a convertirse en una herramienta necesaria en la praxis formativa y profesional en las diferentes carreras y áreas del saber.

Se comparte con Contreras, González, \& Fuentes, (2011); Contreras, 2017 seguidos por Gellibert, et al., (2021), en que: "las Tecnologías de la Información y la Comunicación son herramientas valiosas en la docencia". A esto se puede añadir que actualmente son extremadamente esenciales para el logro de aprendizajes significativos y sustentables en cualquier carrera o nivel de enseñanza. En este sentido, García et al., (2018), aseveran que estas herramientas didácticas fortalecen la comunicación y la interacción en las diferentes actividades, propiciando una mayor motivación en los estudiantes.

Otras investigaciones precedentes realizadas por George \& Filak, (2009); Li-Ping y Jill, (2009); Park, et, al, (2010), seguidos de Du y Xu, (2010)), se centraron en delimitar la importancia de la aplicación de las Tecnologías de la Información y la Comunicación en los procesos de aprendizaje. Concretamente, han valorado qué tantos aprendizajes obtienen los estudiantes a partir de la aplicación de las Tecnologías de la Información y la Comunicación, analizando si esta praxis basada en las Tecnologías de la Información y la Comunicación, los motivan y los satisfacen o no resulta ser así. Por otra parte logran establecer la relación entre las herramientas tecnológicas usadas y las tareas o actividades realizadas en los cursos.

En esta línea Carrascal, Alvarino \& Díaz, (2009), Mukama, (2010), según Caicedo, \& Rojas, (2014), se agruparon en diferentes estudios para medir los impactos de las Tecnologías de la Información y la Comunicación, en el desempeño del estudiante en una tarea de clase, ejemplo, valoraron cómo construyen conocimiento en tareas académicas apoyadas en herramientas tecnológicas: tanto en forma asincrónicas como sincrónicas. De igual manera otras investigaciones han estado dirigidas a evaluar el efecto negativo de la introducción de las Tecnologías de la Información y la Comunicación en el desempeño académico de los estudiantes.

Derivado de todo el análisis realizado se coincide con Caicedo, \& Rojas, (2014), al explicar que:" para facilitar la construcción de aprendizajes significativos no es suficiente 
incluir herramientas tecnológicas a las prácticas pedagógicas, sino que es necesario incorporarlas desde una clara comprensión de los factores que intervienen en el aprendizaje, así como de los contenidos que se van a enseñar, de los objetivos de aprendizaje y de las necesidades de los estudiantes". En otras palabras se requiere organizar el proceso de enseñanza-aprendizaje coherentemente articulado a las necesidades y potencialidades existentes en los grupos que aprenden, conjuntamente con las competencias y habilidades de los docentes que enseñan.

Se concuerda además con los autores mencionados al explicar que el uso de las Tecnologías de la Información y la Comunicación en los procesos de enseñanza y aprendizaje, no solamente requiere de la apropiación por parte del estudiante, sino también del profesor, quien debe ajustar sus actividades y utilizar dichas herramientas al servicio de la construcción de conocimientos específicos en un área determinada Caicedo, Montes y Ochoa Angrino, (2013); Chávez y Caicedo, (en prensa).

Según Carneiro, et al., (2021), "la incorporación de las Tecnologías de la Información y la Comunicación en la educación ha abierto grandes posibilidades para mejorar los procesos de enseñanza y de aprendizaje". Apunta además que, no es suficiente con dotar a las escuelas de computadores, sino que es necesario también un cambio en la organización de las escuelas y en las competencias digitales de los profesores.

Lo referido está ligado con lo destacado por Chávez y Caicedo, (en prensa), al explicar que: "la integración de las Tecnologías de la Información y la Comunicación, a las prácticas educativas, no solo depende de escoger la herramienta más apropiada, sino que también obedece a las competencias del profesor para diseñar tareas estructuradas, que den a los estudiantes la información necesaria y suficiente sobre las metas, el proceso y las demandas de aprendizaje," unido por supuesto a las pautas y orientaciones que les ayuden en el desarrollo de las estrategias más idóneas para el proceso de aprendizaje y el logro de los objetivos previsto. Compartiéndose con Belén, (2010), que en muchas ocasiones influye también la escasa preparación de los docentes para el manejo de las Tecnologías de la Información y la Comunicación, presentándose justo ahí un gran desafío

En el caso de Ecuador se concierta con Hidalgo, et al., (2018), al plantear que se han dado pasos de avances importantes en cuanto a desarrollo tecnológico y su introducción en el contexto educativo. Enfatizan los autores que Ecuador, se encuentra enmarcado en el camino de la tecnología y los avances en las comunicaciones como un mecanismo de modernización del país, es por ello que la Secretaria Nacional de Ciencia y Tecnología (SENACYT), promueva dentro de sus políticas y prioridades todo lo relacionado con la vinculación, cooperación e integración de Ecuador a las redes mundiales de la información, suscitando el progreso de acciones encaminadas a aperturar soluciones científicotecnológicas en zonas rurales, en el contexto de la medicina, la educación y los servicios en línea. 
En este sentido somos del criterio que para lograr lo antepuesto se requiere optimizar las infraestructuras de acceso, ampliar la cultura social en cuanto al uso de la tecnología y reformular los currículos universitarios de forma tal que se involucre en ello toda la sociedad y a todos los niveles educativos con especial atención las universidades Un aporte muy importante emanado de la investigación de los autores precedentes está en la identificación que establecen en cuanto a la importancia de propiciar en las Universidades desde el propio currículo universitario, el uso de las Tecnologías de la Información y la Comunicación, subrayan que esta es la única vía para garantizar la competitividad en las universidades modernas

\section{De lo General a lo Particular.}

Según Espindola, et, al (2012), "la Estadística es una rama de la matemática aplicada, cuyo uso se manifiesta en la recopilación, procesamiento y análisis de la información relacionada con diferentes datos, los cuales permiten su comprobación y fiabilidad práctica”. A esto se debe agregar que al pertenecer al grupo de asignaturas que integran el perfil aplicativoinvestigativo de cualquier carrera, es de gran importancia ya que facilita la vinculación de la teoría con la práctica y la comprobabilidad de los datos obtenidos en las diferentes investigaciones.

Siguiendo esta línea autores como Rojas, (2002), Carmona, (2004), seguidos por Ruiz (2007), Faustino (2012) \& Faustino, \& Pérez, (2013), desde hace varios años han venido sistematizando e investigando sobre la importancia de la utilización de los recursos tecnológicos en el proceso de enseñanza-aprendizaje en los diferentes niveles de enseñanza, con especial interés en la Educación Superior.

De las investigaciones precedentes se deduce que las Tecnologías de la Información y la Comunicación y su aplicación en el proceso de enseñanza - aprendizaje de la Estadística en la Educación Superior, en su generalidad han tenido un impacto favorable, revelándose que se trata de una opción viable y efectiva para el logro de una mejor calidad en el proceso de enseñanza-aprendizaje de esta asignatura. Su introducción favorece el desarrollo de habilidades y destrezas necesarias para el análisis, interpretación de los resultados investigativos y por consiguiente asegura un aprendizaje mucho más significativo. Sin embargo, este proceso didáctico- metodológico ha tenido que transitar por diferentes miradas y caminos, los cuales no siempre han resultado propicios.

Es por ello importante considerar que en su conjunto en los diferentes países en el contexto de la enseñanza-aprendizaje de la Estadística en la Educación Superior se han encontrado diferentes barreras, las cuales van desde la falta de recursos tecnológicos, hasta aquellas relacionadas directamente con las competencias y habilidades tanto de los Docentes - Investigadores, al presentar limitaciones en el manejo adecuado de las metodología didácticas para la enseñanza y aplicabilidad de la Estadística, hasta las que están presentes en los estudiantes que aprenden.

Se concierta con Faustino, \& Pérez, (2013), al señalar que: "en muchas ocasiones los estudiantes universitarios no poseen las habilidades cognitivas suficientes para la 
realización de las acciones que exige el proceso de construcción del conocimiento teórico científico, de forma que puedan articularlo con el conocimiento investigativo desde la sistematización de métodos estadísticos y su utilización en la clasificación, descripción, análisis, presentación e interpretación de la información obtenida a través de técnicas propias de la investigación, que contribuyan a la solución de problemas profesionales.."; como es lógico pensar esto influye grandemente en el desarrollo de sus competencias profesionales provocando la necesidad de encontrar alternativas que ayuden a solucionar esta problemática.

Lo expresado hasta el momento nos reafirma dos ideas fundamentales, la primera está relacionada con la importancia del tema objeto de estudio y la segunda se resume en que la diversidad de investigaciones realizadas sobre este tema muestran los beneficios de la aplicación de las Tecnologías de la Información y la Comunicación en el proceso docente educativo, pero paradójicamente en cuanto al proceso de enseñanza-aprendizaje de la Estadística, en la Educación Superior aun no se alcanzan por diferentes causas los niveles deseados y necesarios a lograr. Dentro de estas las limitaciones se encuentra la accesibilidad, limitaciones en el manejo de las herramientas tecnológicas y en la propia aplicación de la Estadística, así como la falta de creatividad pro parte de los docentes para aplicar esta novedosa herramienta en el proceso de enseñanza-aprendizaje.

\section{Metodología}

La investigación siguió una metodología descriptiva, mediante la Revisión Sistemática de Literatura, (RSL), acompañada del empleo de métodos teóricos, tales como: el histórico-lógico, el analítico - sintético, inductivo - deductivo y la revisión documental, los cuales permitieron revisar 22 fuentes de diferentes bases de datos relacionados con el problema objeto de estudio. El rango de tiempo establecido comprendió las investigaciones realizadas entre el 2000 y el 2021.Determinandose 5 investigaciones altamente significativas

Para establecer los artículos y circunscribir la población en la revisión realizada se establecieron como palabras claves: Tecnología de la Información y Comunicación y Estadística (TIC-E), quedando estos instaurados como los conectores AND identificados para la ejecución de la búsqueda en las diferentes bases de datos.

La estrategia de búsqueda desarrollada siguió tres etapas: planificación, análisis y resultados, lo cual permitió cumplir con los objetivos y calidad de la investigación.

Criterios de inclusión

1. Tipo de estudios: Estudios de revisiones teóricas o experimentales que hayan sistematizado o desarrollado teorías y metodologías en relación con la utilización de las Tecnologías de la Información y la Comunicación en la enseñanza de la Estadística en la Educación Superior.

2. Tipo de participantes: investigadores, docentes, directivos, profesionales vinculados con los procesos de enseñanza-aprendizaje. 
3. Tipo de resultados: Revisiones Bibliográficas, estudios Descriptivos, Documentales, Metodologías, Programas, Estrategias de intervención relacionadas con el problema objeto de estudio.

Criterios de exclusión

1. Estudios o investigaciones realizadas en un rango de tiempo diferente al de la investigación.

2. Artículo en idioma diferente del español o inglés.

3. Otros estudios que por su tema o resultados no aporten elementos significativos para la investigación.

Evaluación de la calidad

La calidad se determinó mediante el seguimiento riguroso de los criterios de inclusión y exclusión establecidos previamente.

Fuentes y técnicas de recopilación de información de análisis de datos

Para la búsqueda de los antecedentes primarios de investigación se utilizaron buscadores de información científica especializada, tales como: Google Académico, Latindex, Dialnet, Scopus, entre otras.

Tabla No 1. Resultados de la búsqueda realizada Artículos referentes para la investigación entre los años (2000- 2021).

\begin{tabular}{|c|c|c|c|c|}
\hline Nombre del autor & Titulo & Año & $\begin{array}{l}\text { Base de publicación y } \\
\text { tema }\end{array}$ & Aportes \\
\hline Belén, AM., & $\begin{array}{l}\text { Formación } \\
\text { docente en } \\
\text { TICS. ¿Están } \\
\text { los docentes } \\
\text { preparados para } \\
\text { la (r) evolución } \\
\text { tic? }\end{array}$ & 2010 & 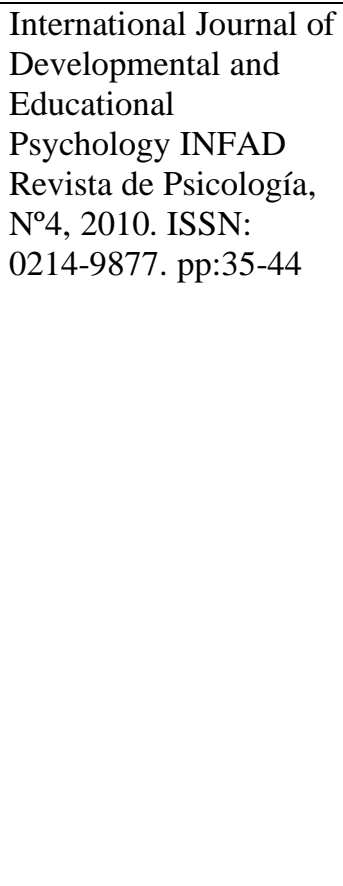 & $\begin{array}{l}\text { El trabajo hacer un } \\
\text { breve recorrido por } \\
\text { los mayores } \\
\text { problemas } \\
\text { encuentran que } \\
\text { profesores para la } \\
\text { inclusión de las TIC } \\
\text { en el aula, vinculados } \\
\text { en su mayor parte a } \\
\text { una formación escasa } \\
\text { o inadecuada. En } \\
\text { segundo lugar, señala } \\
\text { algunos aspectos que } \\
\text { se deberían tener en } \\
\text { cuenta en el diseño de } \\
\text { la formación docente, } \\
\text { destacando la } \\
\text { importancia } \\
\text { conocimiento del } \\
\text { metodológico- } \\
\text { pedagógico de las } \\
\text { TIC. }\end{array}$ \\
\hline $\begin{array}{l}\text { Caicedo, A M., \& } \\
\text { Rojas, T., }\end{array}$ & $\begin{array}{l}\text { Creencias, } \\
\text { Conocimientos } \\
\text { y Usos de las } \\
\text { TIC de los }\end{array}$ & (2014), & $\begin{array}{l}\text { Educación y } \\
\text { Educadores, vol. 17, } \\
\text { núm. 3, pp. 517-533 } \\
\text { Universidad de La }\end{array}$ & $\begin{array}{l}\text { Investigó las } \\
\text { creencias } \\
\text { conocimientos que } \\
\text { tienen los profesores } \\
\text { acerca de la aplicación }\end{array}$ \\
\hline
\end{tabular}


profesores universitarios

Hidalgo, B. G., Castillo, B. F., Hidalgo, D. P., \& Hidalgo, I. M.
Gellibert, SJ., (1) Zapata, SE., Díaz, PJ

\begin{abstract}
La percepción de la incursión de las TIC en el aula desde la perspectiva de los estudiantes universitarios
\end{abstract}

Las TIC en la educación superior durante la pandemia de la COVID-19.

\section{(2018)}

INNOVA Research Journal, 3(6), 53-65. https://doi.org/10.3389 0/innova.v3.n6.2018.5 49

Revista Sinapsis. Vol 1, Nro 19 de las TIC en la enseñanza, y cuáles son los propósitos para su uso. Resultados obtenidos. Aunque las creencias son positivas $y$ podrían favorecer la apropiación de las TIC a sus prácticas educativas, se discuten las implicaciones del conocimiento de los profesores y los usos propuestos, desde los propósitos educativos con que las usan.

La investigación tuvo como objetivo:

Determinar el uso y aplicación de las TIC en el aula y su influencia en el proceso enseñanza aprendizaje desde la percepción de los estudiantes y docentes universitarios de pregrado. Resultados Un porcentaje muy bajo de los docentes usan las TIC en el proceso enseñanza aprendizaje de la bioestadistica, $\quad \sin$ embargo los estudiantes creen firmemente que la incursión de las mismas darán un impacto positivo en el proceso educativo, como se ha comprobado la hipótesis planteada mediante estadística.

El objetivo de la investigación fue analizar el impacto del uso de las TIC en la modalidad de clases en línea de la Universidad de Guayaquil en tiempo de pandemia de la COVID-19 en

Ecuador.

Determinándose TIC 


$\begin{array}{lrll}\text { Carneiro, } \quad \text { R., } & \text { Los desafíos de } & 2021 & \text { Colección Metas } \\ \text { Toscano, JC., Diáz, } & \text { las TIC para el } & & \text { Educativas 2021 } \\ \text { T., } & & \text { Funbio } \\ & \text { educativo } & \text { ISBN: 978-84-7666- } \\ & & 197-0\end{array}$

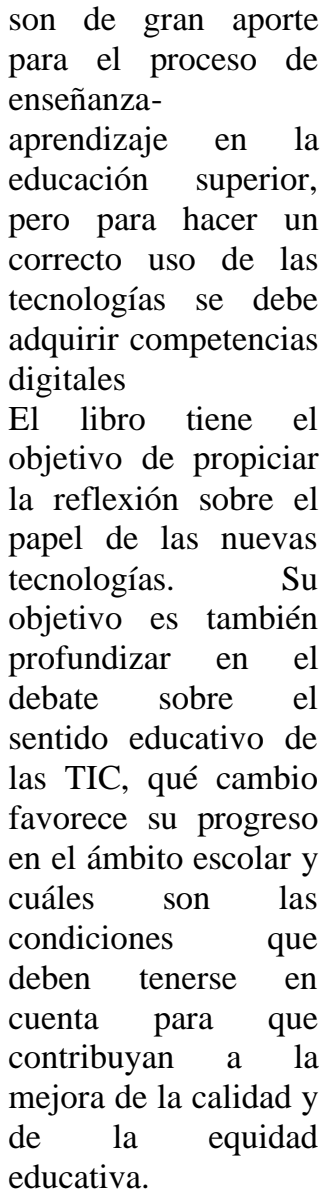
proceso de educación superior, pero para hacer un correcto uso de las tecnologías se debe adquirir competencias digitales El libro tiene el objetivo de propiciar la reflexión sobre el papel de las nuevas objetivo es también profundizar en el debate sobre el sentido educativo de las TIC, qué cambio favorece su progreso en el ámbito escolar y cuáles son las condiciones que deben tenerse en contribuyan a la mejora de la calidad y educativa.

\section{Resultados}

Derivado del análisis y consulta de las fuentes primarias de información se derivan los siguientes resultados:

1. Se confirma la alta utilidad que tiene en el proceso de enseñanza-aprendizaje en la Educación Superior el empleo de las Tecnologías de la Información y la Comunicación con particular interés en la enseñanza de la Estadística, en este sentido se coincide con Rojas, (2002), Carmona, (2004), seguidos por Ruiz (2007), Faustino (2012) \& Faustino, \& Pérez, (2013), Caicedo, \& Rojas, (2014), seguidos de Hidalgo, et al., (2018), Carneiro (2021) al destacar su importancia y enfatizar en la necesidad de articular en ello tanto las competencias de los docentes como de los estudiantes. De esto se deriva a nuestro criterio una idea esencial: mientras más se empleen dichas tecnologías en el proceso de enseñanzaaprendizaje y mientras mejor esté preparado el docente para su selección y aplicación mejores resultados y optimización se logrará en el proceso de enseñanza-aprendizaje.

2. Por otra parte es evidente que a pesar de los avances logrados y de las bondades de las Tecnologías de la Información y la Comunicación para el proceso de enseñanza-aprendizaje de la Estadística en el contexto de la Educación Superior, aún se requiere desarrollar alternativas de capacitación direccionadas a los docentes para que perfeccionen su proceso de enseñanza- aprendizaje en 
interacción directa con las Tecnologías de la Información y la Comunicación de forma tal que puedan emplearlas de forma oportuna en la enseñanza de la Estadística y del resto de las asignaturas.

3. Es necesario desarrollar acciones conducentes a la mejoría de la gestión formativa y cultural tanto de los docentes como de los estudiantes en función de alcanzar habilidades metodológicas que les permitan interactuar significativamente mediante la utilización de las Tecnologías de la Información y la Comunicación, coincidiéndose con Belén, (2010), Gellibert, , et al., (2021) y Carneiro, et al., ( 2021)

4. Una solución transformadora para el proceso de enseñanza y aprendizaje de la Estadística y posible a desarrollar también en el contexto ecuatoriano, puede ser la elaboración de estrategias didácticas contentivas de la creación de software interactivo que faciliten la enseñanza-aprendizaje de esta asignatura. Su empleo y generalización como supuestos mediadores didácticos contribuirá a la mejora de la calidad del proceso de enseñanza-aprendizaje en esta materia en la Educación Superior.

5. Es recomendable además que toda propuesta de solución se encamine sobre el desarrollo de cinco fases, mismas que permitirán desde el proceso de Diagnóstico determinar donde se encuentran las principales fortalezas y debilidades tanto en los Docentes como en los estudiantes, seguida de la fase de Diagnóstico y sobre la base de los resultados obtenidos, sería conveniente desarrollar la segunda fase, relacionada con la Planificación y Selección de la estrategia de trabajo a seguir concerniente a la introducción y aplicación de las Tecnologías de la Información y la Comunicación en el proceso de enseñanza- aprendizaje de la Estadística. La tercera fase comprende todo lo relacionado con la Ejecución de dicha estrategia, hasta llegar a la cuarta fase dirigida al proceso de valoración de los resultados obtenidos. Es importante significar que esta fase debe comprender la retroalimentación constante de los avances logrados para finalmente llegar a la generalización de los resultados.

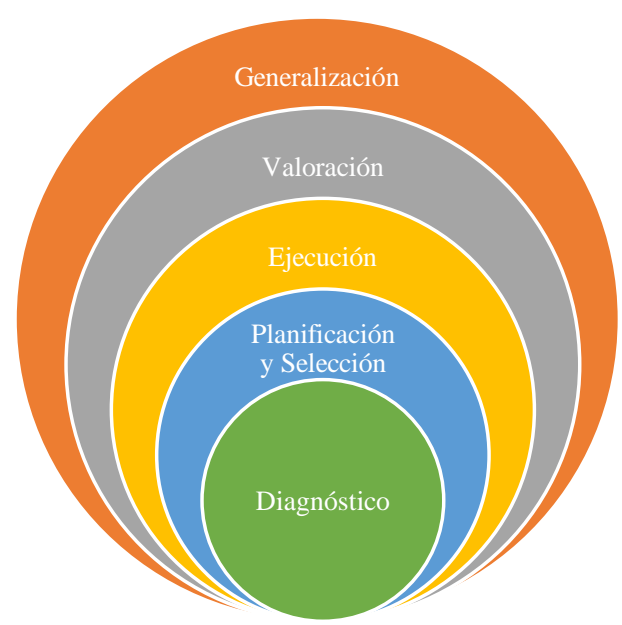

Gráfico No 1. Fases para el desarrollo de la Estrategia de Enseñanza-Aprendizaje Elaborado por: 


\section{Conclusiones}

A modo de conclusión es importante resaltar dos ideas fundamentales:

- La Sistematización de las investigaciones precedentes fundamenta las ventajas de la aplicabilidad de las Tecnologías de la Información y la Comunicación en el proceso de enseñanza-aprendizaje de la Estadística, sin embargo por diferentes causas aún no se logran alcanzar los estándares necesarios y deseados en este ámbito.

- Se requiere implementar estrategias que favorezcan el desarrollo de competencias tanto en los docentes como en los estudiantes para que puedan aplicar las Tecnologías de la Información y la Comunicación en el proceso de enseñanzaaprendizaje de la Estadística, como una alternativa oportuna para el logro de aprendizajes significativos, así como de la generalización de estas.

\section{Referencias Bibliográficas}

Belén , AM., (2010), Formación docente en TICS. ¿Están los docentes preparados para la (r) evolución tic? International Journal of Developmental and Educational Psychology INFAD Revista de Psicología, No4, 2010. ISSN: 0214-9877. pp:3544

Caicedo, A M., \& Rojas, T., (2014), Creencias, Conocimientos y Usos de las TIC de los profesores universitarios Educación y Educadores, vol. 17, núm. 3, pp. 517-533 Universidad de La Sabana Cundinamarca, Colombia

Carmona, M. (2004). Una revisión de las evidencias de fiabilidad y validez de los cuestionarios de actitudes y ansiedad hacia la Estadística. La Habana: Editorial Científico Técnica.

Carrascal, N., Alvarino, G., y Díaz, E., (2009). Estrategias mediadas por TIC para el desarrollo de enfoque de aprendizaje profundo en estudiantes universitarios. Folios, 15, 3-18.

Carneiro, R., Toscano, JC., Diáz, T., (2021), Los desafíos de las TIC para el cambio educativo Colección Metas Educativas. Fundación Santillana ISBN: 978-847666-197-0

Contreras, L., González, K., \& Fuentes, H. (2011). Uso de las TIC y especialmente del blendedlearning en la enseñanza universitaria. Educación y desarrollo social, 151160. https://doi.org/10.18359/reds.898

Contreras, J. L. R. (2017). Importancia de las TIC en enseñanza de las matemáticas. Revista Matemática de la Universidad del Atlántico, 4(2), 1. Recuperado de: http://investigaciones.uniatlantico.edu.co/revistas/index.php/MATUA/article/vie w/1861 
Chávez, J. \& Caicedo, A. M. (en prensa). TIC y argumentación: Análisis de tareas propuestas por docentes universitarios. Estudios pedagógicos.

Du, J. y Xu, J. (2010). The quality of online discussion reported by graduate students. The Quarterly Review of Distance Education, 11 (1), 13-24.

Espindola, A., Gutiérrez, M., Castellanos, X., Yordi , I., \& Miranda, M., (2012). Estrategia didáctica para la dinámica del proceso docente educativo de la Matemática en la especialidad Bioestadística Didactic strategy for the dynamic of the teaching- educative process of Mathematics, within Biostatistics specialty, 12(2), 347-359.

Retrieved from http://scielo.sld.cu/pdf/hmc/v12n2/hmc15212.pdf

Faustino, A. (2012). Resultados de la aplicación de las Tecnologías de Información y Comunicación para el proceso de enseñanza aprendizaje de la asignatura de Estadística en la Educación Superior Angolana. La Habana: Editorial Félix Varela.

Faustino, A., Pérez, S., (2013). Utilización de las TIC en la enseñanza de la Estadística en la Educación Superior Angolana. Prisma Social, Núm. 11, pp. 0-31 IS+D Fundación para la Investigación Social Avanzada. Las Matas, España

García, M., Reyes, J., \& Godínez, G. (2018). Las TIC en la educación superior, innovaciones $\mathrm{y}$ retos / The ICT in higher education, innovations and challenges.RICSH Revista Iberoamericana de las Ciencias Sociales y Humanísticas, 6(12), 17. https://doi.org/10.23913/ricsh.v6i12.135

George, J., \& Filak, V.,(2009). Blended learning in the visual communications classroom: Student reflections on multimedia course. Electronic Journal of E-Learning, 7 (3), 247-256

Gellibert, SJ., (1) Zapata, SE., Díaz, PJ., (2021) Las TIC en la educación superior durante la pandemia de la COVID-19. Revista Sinapsis. Vol 1, Nro 19.

Hidalgo, B. G., Castillo, B. F., Hidalgo, D. P., \& Hidalgo, I. M. (2018). La percepcion de la incursión de las TIC en el aula desde la perspectiva de los estudiantes universitarios. INNOVA Research Journal, 3(6), 53-65. https://doi.org/10.33890/innova.v3.n6.2018.549

Li-Ping, T. \& Jill, M., (2009). Student's perception of teaching technologies, application of technologies, and academic performance. Computers and Education, 53, 12411255.

Mukama, E. (2010). Strategizing computer-supported collaborative learning toward knowledge building. International Journal of Educational Research, 49, 1-9. 
Park, C., Crocker, C., Nussey, J., Springate, J. y Hutchings, D. (2010). Evaluation of a teaching tool - wiki - in online graduate education. Journal of Information Systems Education, 21 (3), 313-321.

Rojas, A., (2002). "Introducción al estudio de los medios de enseñanza". Tesis Doctoral, Centro de Estudios Pedagógicos de Educación Superior Manuel F. Gran, Santiago de Cuba.

Ruiz, F., (2007). Modelos didácticos para la enseñanza de las ciencias naturales. Revista Latinoamericana de Estudios Educativos.

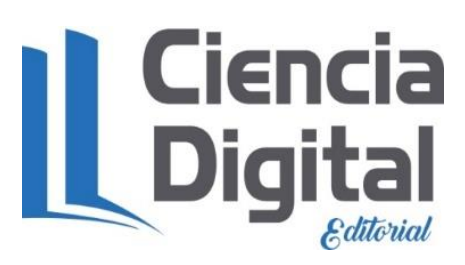




\section{PARA CITAR EL ARTÍCULO INDEXADO.}

Gavilanes Sagñay, M. A. ., Gavilanes-Sagnay, F., \& Yanza Chávez, W. G. (2021). Las Tecnologías de la Información y la Comunicación: Alternativa para la enseñanza de la Estadística Inferencial en la Educación Superior. AlfaPublicaciones, 3(3.1), 42-56. https://doi.org/10.33262/ap.v3i3.1.75

\section{Ciencia \\ LDigitalal}

El artículo que se publica es de exclusiva responsabilidad de los autores y no necesariamente reflejan el pensamiento de la Revista Alfa Publicaciones.

El artículo queda en propiedad de la revista y, por tanto, su publicación parcial y/o total en otro medio tiene que ser autorizado por el director de la Revista Alfa Publicaciones.
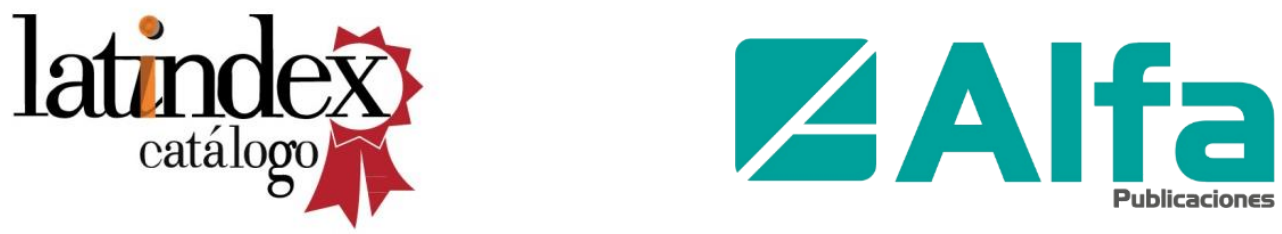